\title{
Laparoscopic Deroofing and Excision of a Large Left Renal Cyst Mimicking Chest Infection: Case Report
}

\author{
Augustine O. Takure, Sikiru A. Adebayo and Gabriel O. Ogun
}

\section{ABSTRACT}

\begin{abstract}
Background: The routine use of abdominal ultrasonography and computed tomography scan has increased the detection of asymptomatic renal cysts. Laparoscopy is usually suitable for treating large symptomatic renal cysts.
\end{abstract}

Objective: To report a case of laparoscopic treatment of a man with large left renal cyst mimicking chest infection.

Case presentation: A 75-year-old man, known hypertensive who had empirical treatment for chest infection without resolution of symptoms of cough and chest pain. He was on treatment for storage and voiding lower urinary tract symptoms with $10 \mathrm{mg}$ alfuzocin. Abdominal ultrasound and computed tomography scan confirmed a large left renal cortical cyst that measured $380 \mathrm{mls}$. He subsequently had transperitoneal laparoscopic deroofing and excision of the renal cyst with operation finding of $300 \mathrm{mls}$ of straw coloured fluid excluding spillage. The immediate postoperative period was uneventful, and he was discharged home in a stable condition on the second day. The aspirate yielded no growth while the cytology report of cystic fluid was acute-on-chronic inflammation. Histology was reported as chronic pyelonephritis with cystic degeneration.

Conclusion: Large renal cyst should be considered as differential diagnosis of unresolved chest infection and is safely treated by laparoscopic deroofing and excision.

Keywords: Chest infection, large renal cyst, laparoscopic deroofing and excision.
Submitted: September 5, 2021

Published: October 7, 2021

ISSN: 2593-8339

DOI: $10.24018 /$ ejmed.2021.3.5.1046

\section{A. O. Takure*}

Department of Surgery, College of Medicine, University of Ibadan and University College Hospital, Ibadan, Nigeria.

(e-mail: aotakure@com.ui.edu.ng)

S. A. Adebayo

Department of Surgery, College of Medicine, University of Ibadan and University College Hospital, Ibadan, Nigeria.

(e-mail: sikobay@gmail.com)

G. O. Ogun

Department of Pathology, College of

Medicine, University of Ibadan and University College Hospital, Ibadan, Nigeria.

(e-mail: olabiyiogun@gmail.com)

*Corresponding Author

\section{INTRODUCTION}

The widespread availability and use of abdominal diagnostic ultrasonography and computed tomography scan led to the increase in the detection of asymptomatic renal cysts [1]. Computed tomography scan and magnetic resonance were employed in the classification of renal cysts into simple or complex based on the thickness of the cyst wall, presence of septa, loculations, internal echoes, and contrast enhancement [2]-[4]. This is well described using the Bosniak classification of simple renal cyst into category I to IV, where categories I and II are simple cysts while III and IV are complex cysts. However, category IIF, III and IV have $5 \%, 50 \%$ and $75 \%$ chances of malignancy respectively [2].

Asymptomatic simple renal cysts are usually observed because of the slow increase in size of $1.6 \mathrm{~mm}$ per year [3]. The complex cysts require more aggressive evaluation and often require surgical intervention such as open surgery, laparoscopic partial or total nephrectomy depending on whether the kidney is solitary or both kidneys are present with a normal contra-lateral one.

The treatment of simple renal cyst is reserved for the symptomatic cyst that present with pain due to infection or haemorrhage or those that are complicated by hydronephrosis and hypertension. Such treatment includes ultrasound guided aspiration with or without sclerotherapy, open surgical or laparoscopic cyst deroofing, unroofing, decortication or excision [5], [6].

The aspiration of simple renal cyst with or without sclerotherapy is fraught with a recurrence rate as high as $82 \%$ compared to laparoscopic treatment with a $5 \%$ recurrence rate [6].

To our knowledge, this is the first case report of unresolved chest infection due to a large left cortical renal cyst that was successfully treated by laparoscopic deroofing and excision.

\section{CASE PRESEntation}

A 75-year-old male, retired factory worker who was a known hypertensive heart disease patient with a significant history of alcohol ingestion and cigarette smoking which he stopped at the commencement of treatment for his hypertension. He was admitted into the medical ward on account of fever and cough that was suggestive of a chest infection. He was on evaluation for lower urinary tract symptoms.

Examination revealed a body mass index of $30 \mathrm{~kg} / \mathrm{m}^{2}$ and blood pressure of $140 / 80 \mathrm{mmHg}$. The chest was clinically 
clear. The left kidney was bimanually palpable and measured about $10 \mathrm{~cm}$ by $8 \mathrm{~cm}$. The prostate gland was moderately enlarged, not tender, firm, and smooth.

The urine microscopy yielded no growth. The serum prostate specific antigen was $5.7 \mathrm{ng} / \mathrm{ml}$ while the transrectal prostate biopsy was reported as nodular hyperplasia. The complete blood count and serum electrolyte, urea and creatinine were within normal limit. The chest radiograph showed no focal parenchymal lesion. The abdominal ultrasound scan showed a large left renal cyst with an estimated volume of $380 \mathrm{mls}$ (Fig. 1).

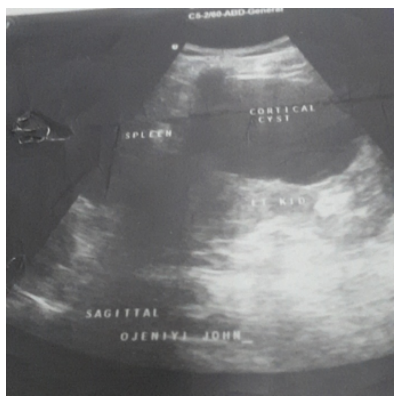

Fig. 1. Abdominal ultrasound showing large left upper renal pole cortical cyst approximately $380 \mathrm{mls}$.

The computed tomography scan in both transverse (Fig. 2a) and corona (Fig. 2b) views showed the large left cortical cyst.
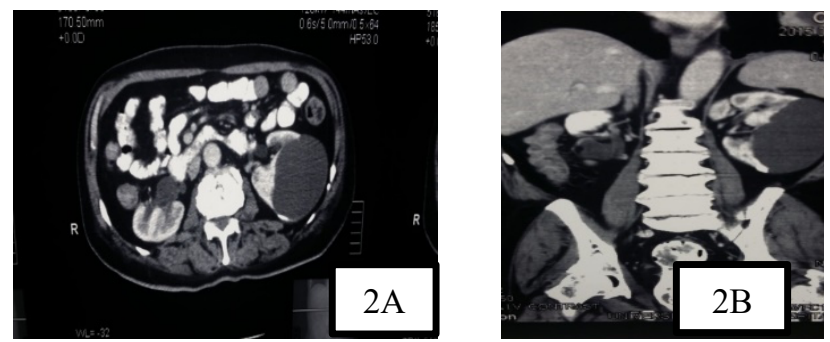

Fig. 2A. Transverse view of CT scanshowing large left cortical cyst with thick wall. Fig. 2B. CT scan corona view showing large left cortical cyst.

He had a transperitoneal laparoscopy with operation finding of large left renal cortical cyst located in the upper pole (Fig. 3) and spillage of straw-colored fluid (Fig. 4).

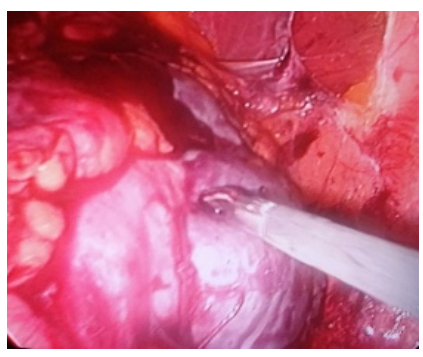

Fig. 3. Large left renal cyst.

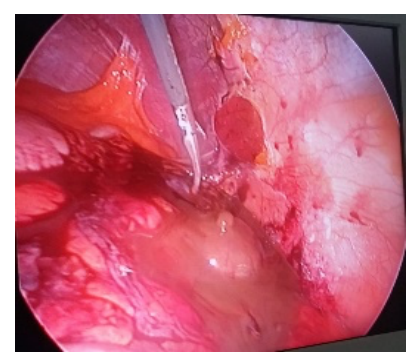

Fig. 4. Spillage of straw colored fluid from the left renal cyst.
$300 \mathrm{mls}$ of fluid was aspirated excluding the spillage (Fig. 5). He subsequently had laparoscopic deroofing with excision of the cyst wall (Fig. 6).

The postoperative period was uneventful, and he was discharged home on the $2^{\text {nd }}$ day after surgery. The chest symptoms subsided completely after the surgery. The laparoscopic fluid aspirate yielded no growth while the cytopathology of cyst fluid revealed acute-on-chronic inflammation. The histology of the cyst wall was chronic pyelonephritis with cystic degeneration (Fig. 7).

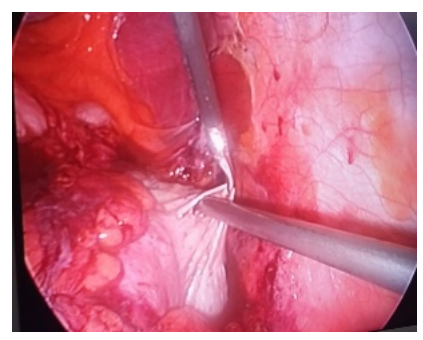

Fig. 5. The aspiration of renal cyst.

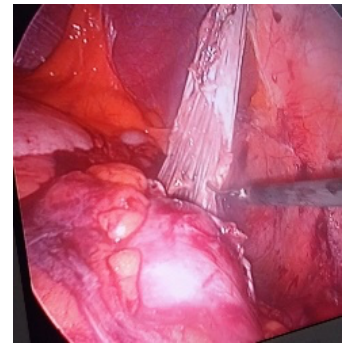

Fig. 6. The excision of the left left renal cyst wall.

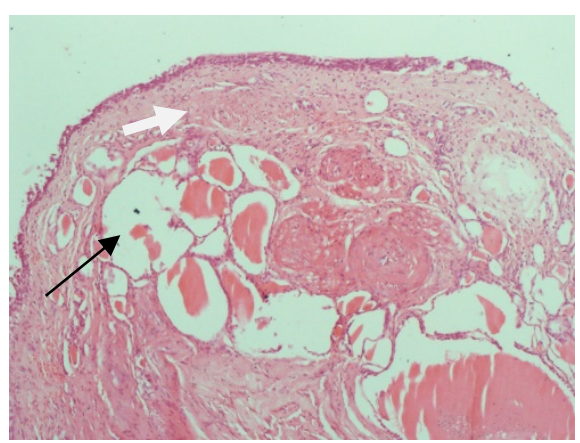

Fig. 7. This slide shows the histology of the excised renal cyst; black arrow is cystic degeneration and white arrow is mononuclear cells.

\section{DISCUSSION}

The most common space occupying lesion of the kidneys are cysts with the prevalence rate that varies from $4.2 \%$ to $10.7 \%$ [7], [8]. Most simple renal cysts are cortical, unilateral, and solitary with well-defined features as in this patient [1], [7], [11].

Currently, the renal cyst is classified based on Bosniak ultrasonographic and computed tomographic features into simple or complex cyst. These features are the round or oval cystic shape, wall thickness (either thin wall or thick wall), septa (e) within the cyst, internal echoes, or posterior enhancement. Categories I and II are simple cysts with no risk of malignancy while category IIF has a 5\% risk of malignancy. Category III is probably malignant and category IV frankly malignant carries a $50 \%$ and $75 \%$ malignancy rates respectively [2], [3]. The patient in this report was classified as Bosniak II because of the posterior wall enhancement with no evidence of malignancy from the cytology and histology.

Our patient was 75 years old which is in tanderm with reports that renal cysts occur with the increase in age and autopsy studies confirmed that $50 \%$ of men over 50 years of age have one or more renal cyst [1], [4], [8]. Both cigarette smoking and hypertension present in this patient are known risk factors for the development of renal cyst [7].

The clinical features of cough and chest infection in this patient were highly suggestive of chest infection. However, the symptoms persisted despite appropriate antibiotics. This patient was seen before the Covid-19 pandemic, otherwise this would been considered as a differential diagnosis of the persistent chest infection. The abdominal ultrasonography was requested to evaluate the intercurrent lower urinary tract symptoms and this revealed the large left renal cyst. Further evaluation with computed tomography characterized the cyst 
and confirmed the diagnosis. The location of the cyst in the upper pole of the left kidney and the histologic report of cystic wall as chronic pyelonephritis with cystic degeneration may explain the diaphragmatic irritation and may explain the cyst mimicking the chest infection. Mathiew et al. [12], had reported a large renal cyst causing gastric symptoms.

This patient underwent transperitoneal laparoscopic deroofing and excision of the cyst which is the commoner laparoscopic approach in the treatment of simple symptomatic renal cyst. Although, retroperitoneal approach is also feasible with comparable outcome. The advantage of laparoscopic treatment is the reduced recurrence rate as low as $5 \%$ compared to ultrasound guided renal cyst aspiration with or without sclerotherapy that has a recurrence rate up to $82 \%$ [4], [6], [12], [13].

\section{CONClusion}

In conclusion, in the absence of Covid-19 infection, any patient who present with persistent unresolved chest infection should have an abdominal ultrasound or CT scan to exclude the presence of large simple renal cyst. The outcome of treatment is excellent following laparoscopic deroofing and excision.

\section{ACKNOWLEDGMENT}

We thank the theatre nurses and the staff of biomedical instrument section of the hospital for their support during the procedure.

\section{REFERENCES}

[1] G. A. Eknoyan, "A clinical view of simple and complex renal cysts," $J$ Am Soc Nephrol 2009;20(9):1874-6.

[2] M. A. Bosniak, "The current radiological approach to renal cysts," Radio 158, 1-10, 1986.

[3] T. M. Weber, "Sonography of benign renal cystic disease," Ultras Clin $1,15-24,2006$.

[4] T. F. Whelan, "Guidelines on the management of renal cyst disease," Can Urol Assoc J 4(2), 98-99, 2010.

[5] M. M. Agarwal and A. K. Hemal, "Surgical management of renal cystic disease," Cur Urol Rep 12(1), 3-10, 2011.

[6] A. A. Okeke, A. Mitchelmore and F. Keeley, "A comparison of aspiration of aspiration and sclerotherapy with laparoscopic de-roofing in the management of symptomatic simple renal cysts," BJU Int 92, 610, 2003.

[7] C. C. Chang, J. Y. Kuo, W. L. Chan, K. K. Chen and L. S. Chang, "Prevalence and clinical characteristics of simple renal cyst," J Clin Med Assoc 70 (11), 486-491, 2007.

[8] A. A. Mosharafa, "Prevalence of renal cysts in a Middle Eastern population: an evaluation of characteristics and risk factors," BJU Int $1(6), 736-8,2008$.

[9] M. Bisceglia, C. A. Galliani, C. Senger, C. Stallone and A. Sessna, "Renal cystic diseases: A review," Adv Anat Pathol 13, 26-56, 2006.

[10] J. M. Kissane and M. G. Smith, "Pathology of infancy and childhood, St. Louis" C. V. Mosby Co 521, 1967.

[11] N. Terada, Y. Arai, N. Kinukawa and A. Terai A, "The 10-year natural history of simple renal cysts," Urology 71(7), 7-12, 2008.

[12] J. Mathiew, M. Al-Mohahoon, N. Machado, R. William and K. Rahman, "Laparoscopic deroofing of large renal simple cyst causing gastric symptoms," Sultan Qaboos Niv Med J 7(2), 139-144, 2007.

[13] N. Haque, M. Hossan and M. Uddin, "Outcome of laparoscopic deroofing of renal cyst- our experience," Medicine today 31(2), 114116, 2019. https://doi.org/10.3329/medtoday.v3li2.41954.

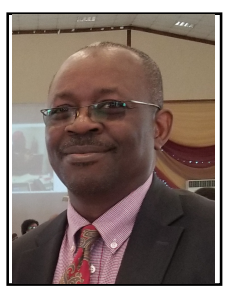

Augustine O. Takure was born 20.8.1964 in Lagos, Nigeria. Graduated with M.B.B.S at the University of Ibadan Nigeria in 1989. Underwent surgery and urology residency training from February 2001 to December 2007 at the Obafemi Awolowo University Teaching Hospital, Ile-Ife, Osun State in Nigeria. Obtained the Fellowship of West African College of Surgeons (FWACS) in 2008. Certificate of training in Renal Transplantation and Endourology July-December 2008 at the Muljibhai Patel Urological Hospital in Nadiad, Gujarat, India. Fellow of International College of Surgeon (FICS) in 2014. Major field of study: general surgery, urology, laparoscopy, and kidney transplantation.

He worked as National Youth Service Corp (NYSC) medical officer at the university health services (1991-1992) in the University of Ibadan (UI). Medical Officer/Senior Medical Officer (1993-Jan. 2001) at UI. Junior Surgery residency training Feb. 2001- Oct. 2004) at the Obafemi Awolowo University Teaching Hospital (OATHC), Ile-Ife, Osun State in Nigeria. Senior urology residency training (Oct. 2004-Dec. 2007) at OAUTHC. Lecturer 1 (Jun. 2008-Oct. 2009) at Ambrose Alli University, Ekpoma, Edo \& Consultant Urologist at Irrua Specialist Hospital, Irrua in Edo State, Nigeria. Hospital Consultant/Associate Lecturer at University College Hospital (UCH) \& College of Medicine, University of Ibadan (COMUI), Oyo State, Nigeria (Nov. 2009-Jan. 2014). Lecturer 1/Senior Lecturer at COMUI (Jan. 2014-date'2021). Honorary Consultant at UCH, Ibadan. Publications covers day case urology, prostate and kidney diseases, renal transplantation, and laparoscopy. Research interests are renal transplantation, laparoscopy, urooncology, male infertility, and reconstructive urology.

Dr. Takure. Membership in Professional societies: Nigeria Medical Association (NMA), Medical and Dental Consultant Association, Nigeria (MDCAN), General Secretary, Association of Urological Surgeons, Nigeria (NAUS), Vice President II, Transplant Association, Nigeria (TAN), Zonal Coordinator, International College of Surgeon (ICS) Nigeria National Section, Deputy National Delegate representing Nigeria in Society Internationale d'Urologie (SIU), European Association of Urology (EAU), and American Urology Association (AUA). 